\title{
KRATTA, a versatile triple telescope array for charged reaction products
}

\author{
J. Łukasik*, P. Pawłowski, A. Budzanowski ${ }^{1}$, B. Czech, I. Skwirczyńska \\ Institute of Nuclear Physics, IFJ-PAN, 31-342 Kraków, Poland
}

J. Brzychczyk, M. Adamczyk, S. Kupny, P. Lasko, Z. Sosin, A. Wieloch

Institute of Physics, Jagiellonian University, 30-059 Kraków, Poland

M. Kiš, Y. Leifels, W. Trautmann

GSI, D-64291 Darmstadt, Germany

\section{Abstract}

A new detection system KRATTA, Kraków Triple Telescope Array, is presented. This versatile, low threshold, broad energy range system has been built to measure the energy, emission angle, and isotopic composition of light charged reaction products. It consists of 38 independent modules which can be arranged in an arbitrary configuration. A single module, covering actively about $4.5 \mathrm{msr}$ of the solid angle at the optimal distance of $40 \mathrm{~cm}$ from the target, consists of three identical, $500 \mu \mathrm{m}$ thick, large area photodiodes, used also for direct detection, and of two CsI $(1500 \mathrm{ppm} \mathrm{Tl})$ crystals of 2.5 and $12.5 \mathrm{~cm}$ length, respectively. All the signals are digitally processed. The lower identification threshold, due to the thickness of the first photodiode, has been reduced to about $2.5 \mathrm{MeV}$ for protons ( $\sim 5 \mu \mathrm{m}$ of $\mathrm{Si}$ equivalent) by applying a pulse shape analysis. The pulse shape analysis allowed also to decompose the complex signals from the middle photodiode into their ionization and scintillation components and to obtain a satisfactory isotopic resolution with a single readout channel. The upper energy limit for protons is about $260 \mathrm{MeV}$. The whole setup is easily portable. It performed very well during the ASY-EOS experiment, conducted in May 2011 at GSI. The structure and performance of the array are described using the results of $\mathrm{Au}+\mathrm{Au}$ collisions at $400 \mathrm{MeV} /$ nucleon obtained in this experiment.

Keywords: charged particle detection, triple telescope, CsI(Tl) scintillator, large area PIN photodiode, low noise preamplifier, pulse shape analysis

\section{Introduction}

Charged-particle detection and identification with isotopic resolution over a large dynamic range in particle type and energy, is mandatory for studies of isotopic effects in heavy-ion reactions. Phenomena related to the isotopic composition of the reaction system and the emitted products have been shown to be useful for exploring the properties of neutron-rich nuclear matter as, e.g., encountered in neutron stars [1,2]. Their importance will increase with the availability of secondary beams of high intensity. Also studies of nuclear structure near the limits of stability require increasingly sophisticated and precise detection systems. A comprehensive overview of the recent and future developments in this field of instrumentation can be found in Ref. [3].

Most of the existing charged particle detectors for the intermediate energy range (up to a few tens or hundreds of $\mathrm{MeV} /$ nucleon) base their identification on the two- or threefold telescope method [4, 5]. In order to provide the lowest

\footnotetext{
${ }^{*}$ Corresponding author.

Email address: jerzy.lukasik@ifj .edu.pl (J. Łukasik)

${ }^{1}$ Deceased.
}

possible identification threshold, the first layer of the telescope is usually made of a gas chamber (e.g. DELF [6], MULTICS [7], FASA [8], INDRA [9], ISiS [10], GARFIELD [11], FIASCO [12]) or of a thin Si detector (e.g. FAUST [13], INDRA [9], LASSA [14], CHIMERA [15], HiRA [16], NIMROD [17], FAZIA [18]). The first, $\triangle \mathrm{E}$, layer is then followed by one or two, thicker, Si detectors or scintillators.

The option with the silicon $\Delta \mathrm{E}$ layer, has the advantage of a better resolution and is easier to handle, but usually results in higher thresholds and is costly. The presented KRATTA modules belong to this class of telescopes, however, they have been optimized to be budget friendly, without loosing the quality of detection. Instead of using the Si detectors of different thickness, they are using three identical, catalog size, photodiodes and two $\mathrm{CsI}(\mathrm{Tl})$ crystals. Thanks to the digital signal processing and the off-line pulse shape analysis, the obtained mass resolutions for light charged particles are very satisfactory in a broad energy range. On one hand, the pulse shape analysis allowed to reduce the energy threshold, resulting from the relatively thick first layer, by a factor of 3 . On the other hand, it allowed to effectively double the thickness of the silicon $\Delta \mathrm{E}$ layer, by combining the ionization components from the first two photodiodes, and consequently, to improve the resolution 
of the first segment of the telescope.

\section{Motivation and requirements}

The main parameters of the KRATTA array have been motivated by the needs of the ASY-EOS experiment [19]. This experiment has been designed to study the density dependence of the nuclear symmetry energy by measuring flows and isotopic compositions of the reaction products from the ${ }^{197} \mathrm{Au}+{ }^{197} \mathrm{Au}$, ${ }^{96} \mathrm{Ru}+{ }^{96} \mathrm{Ru}$, and ${ }^{96} \mathrm{Zr}+{ }^{96} \mathrm{Zr}$ reactions at $400 \mathrm{MeV} /$ nucleon. During the experiment the most relevant products, neutrons and $Z=1$ and 2 particles, have been measured by the LAND [20] detector and the direction and magnitude of the impact vector were estimated using the CHIMERA [15] and ALADIN ToFWall [21] detectors. The KRATTA array has been designed to complement the neutron and hydrogen detection with LAND by measuring the isotopic composition and flow of light charged reaction products up to atomic number $Z \lesssim 7$, and specially, to identify the hydrogen and helium isotopes with a resolution much better than achievable with LAND. The array was placed on the opposite side of the beam with respect to LAND, and covered approximately the same solid angle (160 msr). It has been designed to detect energetic particles emerging from the "mid-rapidity" region of the $400 \mathrm{MeV} /$ nucleon reactions. Modular design, portability, low thresholds (below $3 \mathrm{MeV} /$ nucleon) and high maximum energy $(\sim 260 \mathrm{MeV} /$ nucleon for $p$ and $\alpha)$, allow the array to be used in various configurations and experiments. In particular, it will very well suit the needs of the future cyclotron facility at IFJ-PAN in Kraków, planned for proton beams from 70 to $250 \mathrm{MeV}$. Last, but not least, the KRATTA modules are also compatible with the design of the existing Krakow Forward Wall Detector [22] and can be used for reaching complete coverage of the $2 \pi$ azimuthal angle.

\section{Active elements and geometry}

The modules of KRATTA are composed of three large area HAMAMATSU PIN photodiodes for direct detection [23] and of two CsI(Tl) crystals [24]. The layout and dimensions of these active elements are presented in Fig. 1 and their main characteristics are summarized in Table 1

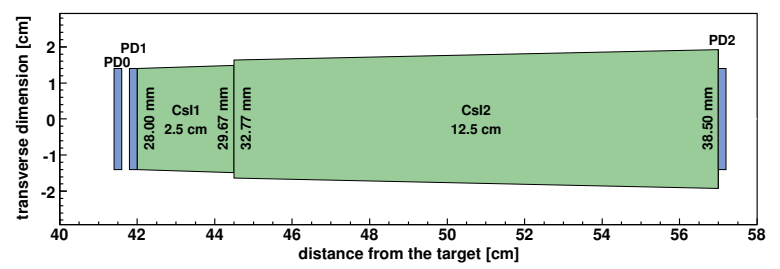

Figure 1: Schematic layout of the active elements.

The first photodiode (PD0 in Fig. 1) serves as a Si $\Delta \mathrm{E}$ detector providing the ionization signal alone. It has been "reverse mount", i.e. the ohmic side towards the incoming particles. The second photodiode (PD1), naturally "reverse mount", works in

\begin{tabular}{ll}
\hline \multicolumn{2}{c}{ Photodiodes [23] $^{\left[28 \times 28 \mathrm{~mm}^{2}\right.}$} \\
Active Area $^{a}$ & $500 \pm 15 \mu \mathrm{m}$ \\
Thickness $^{a}$ & $<3 \mu \mathrm{m}$ \\
Thickness non-uniformity $^{a}$ & $1.5 / 20 \mu \mathrm{m}$ front / rear \\
Dead Layers $^{a}$ & $(111)$ \\
Surface Orientation $^{a}$ & $120-135 \mathrm{~V}$ \\
Full Depletion Voltage $^{b}$ (FD) & $6-16 \mathrm{nA}$, typ. 9 nA \\
Dark Current @ FD $^{b}$ & $190 \pm 3 \mathrm{pF}$ \\
Terminal Capacitance @ FD $^{b}$ & $40 \mathrm{~ns}$ \\
Rise Time (Laser $\delta$ pulse) $^{a}$ &
\end{tabular}

CsI(Tl) Crystals $[24$

$\begin{array}{ll}\text { Tl concentration }{ }^{c} & 1500 \mathrm{ppm} \\ \text { Light output non-uniformity }^{c} & <7 \%\end{array}$

Shape Truncated pyramids

Tolerance $\quad \pm 0.1 \mathrm{~mm}$

\begin{tabular}{lc}
\hline & Wrapping [26] \\
Reflectance $^{a}$ & $>98 \%$ \\
Thickness $^{a}$ & $65 \mu \mathrm{m}$ \\
\hline
\end{tabular}

${ }^{a}$ Values from technical note.

${ }^{b}$ Values from manufacturer's Inspection Sheet.

${ }^{c}$ Nominal values.

Table 1: Main characteristics of the active elements.

a "Single Chip Telescope", SCT [25], configuration and provides a composite signal combined of a direct (ionization) component and of a scintillation component coming from the thin crystal (CsI1). The third photodiode (PD2) reads out the light from the thick crystal (CsI2) and, in addition, provides an ionization signal for particles that punch through the crystal within its active area.

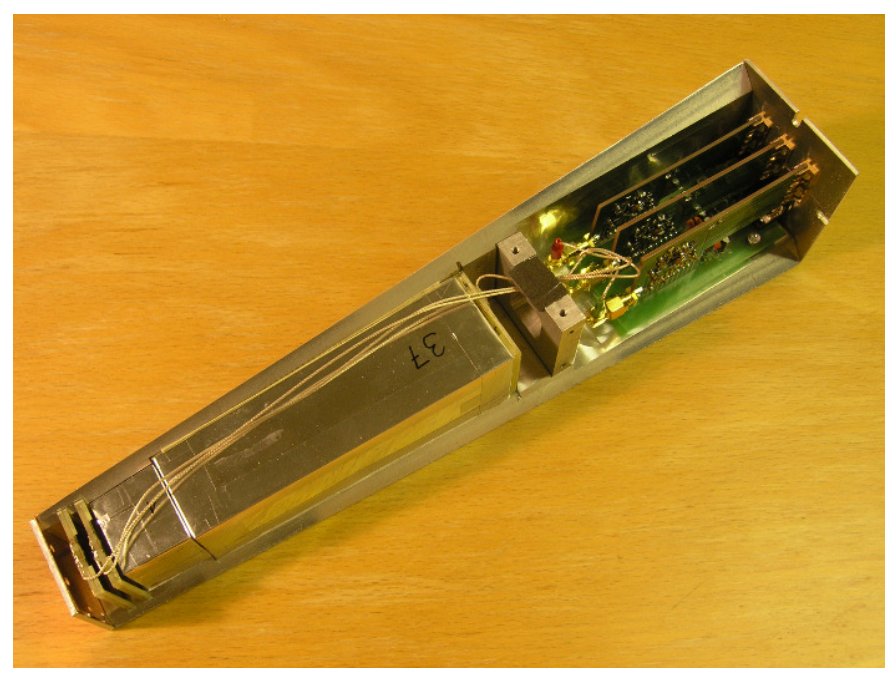

Figure 2: Single module content.

The larger front face of the longer crystal with respect to the rear face of the smaller one (see Fig. 11 permits its use at an about 2 times larger distance from the target, in configuration that completes the missing half of the FWD phoswich 


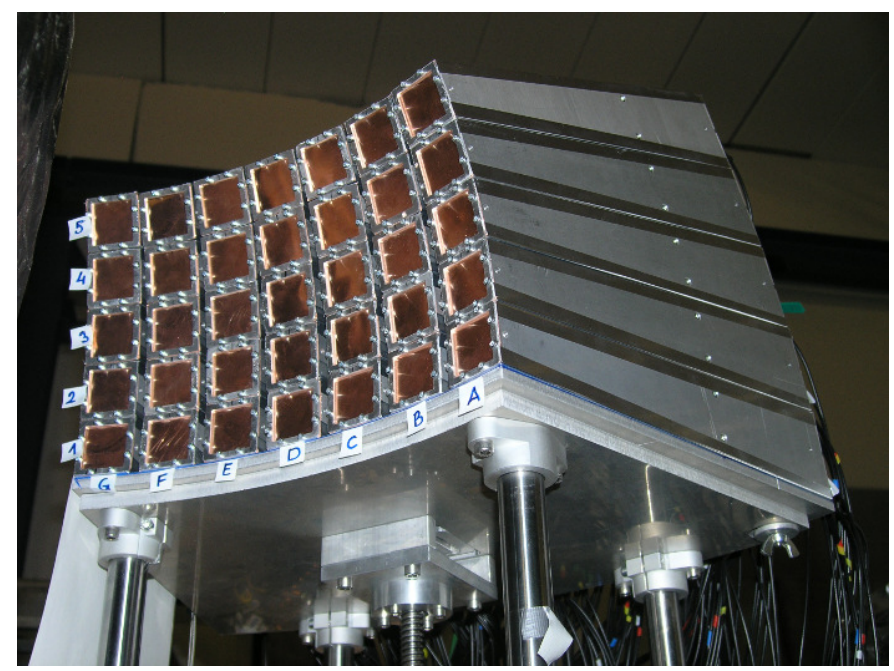

Figure 3: KRATTA in a $7 \times 5$ configuration.

\begin{tabular}{crrr}
\hline Fragment & $\mathrm{E}_{\text {low }}$ & $\mathrm{E}_{\text {int }}$ & $\mathrm{E}_{\text {up }}$ \\
\hline${ }^{1} \mathrm{H}$ & 8.3 & 89.6 & 254.4 \\
${ }^{4} \mathrm{He}$ & 8.3 & 89.4 & 253.9 \\
${ }^{7} \mathrm{Li}$ & 9.5 & 103.6 & 296.5 \\
${ }^{20} \mathrm{Ne}$ & 19.9 & 231.3 & 719.0 \\
${ }^{43} \mathrm{Ca}$ & 26.7 & 339.7 & 1134.2 \\
${ }^{91} \mathrm{Zr}$ & 34.0 & 513.9 & 1911.8 \\
${ }^{197} \mathrm{Au}$ & 38.6 & 775.8 & 3550.9 \\
\hline
\end{tabular}

Table 2: Lower, $\mathrm{E}_{\text {low }}$ and intermediate, $\mathrm{E}_{\text {int }}$, thresholds and upper limits, $\mathrm{E}_{u p}$, for selected species (in $\mathrm{MeV} / \mathrm{u}$ ). The thresholds correspond to the energy losses in $500 \mu \mathrm{m}$ of $\mathrm{Si}$, in $1000 \mu \mathrm{m}$ of $\mathrm{Si}+2.5 \mathrm{~cm}$ of CsI and in $1000 \mu \mathrm{m}$ of $\mathrm{Si}+15 \mathrm{~cm}$ of CsI, respectively. They have been calculated using the ATIMA tables [27].

array [22]. The crystals have been polished and wrapped with a highly reflective ESR [26] foil, except for the front and back windows. The windows have been protected with $6 \mu \mathrm{m}$ Mylar foils. The crystals were optically decoupled. The photodiode chips have been glued onto custom-made PCB frames and put in close optical contact with the crystal windows. The active elements have been placed inside aluminum boxes together with the charge preamplifiers (see Fig. 2). The photodiode frames and the aluminum housing reduced the geometric acceptance of a single module to about $54 \%$. The active solid angle of a module amounts to $4.5 \mathrm{msr}$. The entrance window has been made of a $100 \mu \mathrm{m}$ thick copper foil. During the experiment, 35 modules have been arranged in a $7 \times 5$ array (Fig. 3), all placed at a radial distance of $40 \mathrm{~cm}$ from the target, and operated in air. In a spherical coordinate system, with the origin at the target and the beam axis defining the reference equatorial plane, the columns follow the meridians from the beam level up to about $27^{\circ}$ elevation (latitude) and the rows span the azimuth angles (longitude) between $21^{\circ}$ and $64^{\circ}$ with respect to the beam direction. The energy thresholds resulting from the thicknesses of the consecutive active layers are summarized in Table 2 As will be shown later on, the low threshold can be further reduced by applying a pulse shape analysis to the ionization signals of PD0. The thresholds from Table 2 do not take into account the target, the air and the entrance window present in front of the active layers. In the case of the ASY-EOS experiment, these layers caused increase of the lower threshold, $\mathrm{E}_{\text {low }}$, by about 12.9 MeV/nucleon for protons and $\alpha$ particles (8.6, 1.8 and 2.5 $\mathrm{MeV} /$ nucleon energy losses due to the $350 \mu \mathrm{m}$ Au target, 40 $\mathrm{cm}$ of air and $100 \mu \mathrm{m}$ of $\mathrm{Cu}$, respectively). For low energy experiments, the effects of air and the entrance window can be minimized by performing the measurement in vacuum and removing the $\mathrm{Cu}$ foil or making it thinner.

\section{Electronics and data acquisition}

The main electronics and data acquisition functions are presented schematically in Fig. 4 . The photodiodes (3 per module) have been reverse biased at $120 \mathrm{~V}$, using the in-house made 120-channel, remote controlled, high voltage power supplies. The signal from each photodiode has been integrated with the own-design, low noise, charge preamplifier [28].

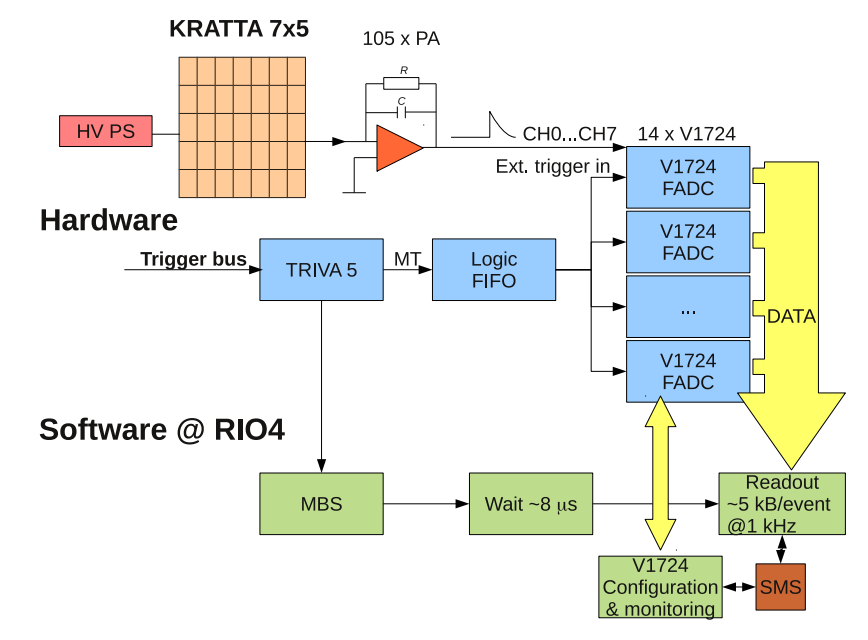

Figure 4: Analog, logical and digital signal flow chart. HV PS - high voltage power supply. PA - charge sensitive preamplifier. V1724 - CAEN digitizers. TRIVA5 - VME Trigger Synchronization Module [30]. MT - master trigger. FIFO - logical FanIn Fan-Out module. RIO4 - VME controller board [31] running LynxOS. MBS - Multi Branch System, a GSI acquisition standard [32]. SMS - Shared Memory Segment.

The preamps were supplied with $\pm 6 \mathrm{~V}$ and their dynamic range spanned about 3.6 V. Three nominal charge gains of the preamplifiers have been used, depending on the azimuthal angle of the module: 44.5, 22.2 and $13.5 \mathrm{mV} / \mathrm{MeV}$, with 1, 2 and $3.3 \mathrm{pF}$ feedback capacitors, respectively. After optional amplification, the signals have been digitized with the $100 \mathrm{MHz}$, 14 bit digitizers [29] and stored for the off line analysis. All logical and digital electronics modules shown in Fig. 4 have been controlled with the RIO4 board [31] within a single VME 
crate. During the experiment, the 14 Flash ADC boards have been triggered with an external trigger split and delivered into each FADC module. The stored waveforms spanned 5.12 or $10.24 \mu \mathrm{s}$ (512 or 1024 time bins), with a $2 \mu$ s pre-trigger enabling a precise baseline estimation. The shorter samples have been sufficient for the first photodiode supplying the fast ionization signal alone. The expected data throughput amounted to about $5 \mathrm{MB} / \mathrm{s}$, assuming $1 \mathrm{kHz}$ single hit rate. The actual data rate did not go beyond this estimate during the experiment. The digitizers have been remotely set up and monitored using a self-developed software. The data flow has been controlled using the standard GSI MBS system [32].

\section{Pulse shape analysis}

The pulse shape analysis has two main purposes in the case of KRATTA data. First of all, it has to enable the decomposition of the signals from the middle photodiode, PD1 (SCT), into the ionization and scintillation components. Secondly, it is used to resolve masses of particles stopped in the first photodiode, PD0, utilizing the relation between the range of a particle and the time characteristics of the induced current signal [33].

The following assumptions have been made to accomplish these two goals. The preamplifier response has been modeled using a simple parallel RC circuit approximation [34]:

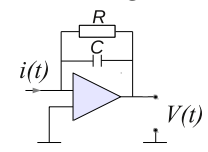

$$
\frac{i(t)}{C}=\frac{d V(t)}{d t}+\frac{V(t)}{R C}
$$

where, $R C$ is the feedback coupling time constant, $i(t)$ is the induced current due to the carrier motion in the photodiode, and $V(t)$ is the measured voltage pulse. This relation assumes an infinite open loop gain, small detector capacitance and a zero rise time of the charge integrator, which is an idealization, but enables an analytical approach. The induced current has been approximated with one direct (ionization), $i_{D}(t)$, and two scintillation, $i_{S k}(t)$, components, all of the same form:

$$
\begin{aligned}
& i_{D}(t)=Q_{D} \frac{e^{-t / \tau_{R D}}-e^{-t / \tau_{F D}}}{\tau_{R D}-\tau_{F D}}, \\
& i_{S k}(t)=Q_{S k} \frac{e^{-t / \tau_{R S}}-e^{-t / \tau_{F k}}}{\tau_{R S}-\tau_{F k}}, \quad k=1,2
\end{aligned}
$$

where $Q$ 's are the induced charges and the $\tau_{R D}, \tau_{R S}$ and the $\tau_{F D}, \tau_{F k}$ are the rise and fall times for the respective component. The assumed shapes attempt to account for both, the complicated actual current pulse shape induced by the electrons and holes drifting in the photodiode [33], and for the instrumental rise time of the preamp. With the assumed preamplifier response (1) and the current shapes (2) and (3), it was possible to obtain the corresponding analytical model shapes of the waveforms, $V(t)=V_{0}+V_{D}(t)+V_{S}(t)$, with:

$$
\begin{aligned}
& V_{D}(t)=R C Q_{D}\left(\frac{e^{-\Delta t / R C} R C}{\left(R C-\tau_{R D}\right)\left(R C-\tau_{F D}\right)}\right. \\
& \left.+\frac{e^{-\Delta t / \tau_{R D}} \tau_{R D}}{\left(\tau_{R D}-R C\right)\left(\tau_{R D}-\tau_{F D}\right)}+\frac{e^{-\Delta t / \tau_{F D}} \tau_{F D}}{\left(\tau_{F D}-\tau_{R D}\right)\left(\tau_{F D}-R C\right)}\right)
\end{aligned}
$$

$$
\begin{aligned}
& V_{S}(t)=R C \sum_{k=1}^{2} Q_{S k}\left(\frac{e^{-\Delta t / R C} R C}{\left(R C-\tau_{R S}\right)\left(R C-\tau_{F k}\right)}\right. \\
& \left.+\frac{e^{-\Delta t / \tau_{R S}} \tau_{R S}}{\left(\tau_{R S}-R C\right)\left(\tau_{R S}-\tau_{F k}\right)}+\frac{e^{-\Delta t / \tau_{F k}} \tau_{F k}}{\left(\tau_{F k}-\tau_{R S}\right)\left(\tau_{F k}-R C\right)}\right)
\end{aligned}
$$

where, for generalization, $V_{0}$ is the baseline and $\Delta t=t-t_{0}$,

\begin{tabular}{|c|c|c|c|}
\hline \multicolumn{2}{|r|}{ Parameter } & \multicolumn{2}{|c|}{ Value } \\
\hline \multicolumn{4}{|c|}{ Ionization } \\
\hline$Q_{D}$ & Amplitude & & fitted \\
\hline$\tau_{R D}$ & Rise time & $\sim 90 \mathrm{~ns}$ & fixed/fitted \\
\hline$\tau_{F D}$ & Fall time & $<300 \mathrm{~ns}$ & fixed/fitted \\
\hline \multicolumn{4}{|c|}{ Scintillation } \\
\hline$Q_{S 1}$ & Fast component amplitude & & fitted \\
\hline$Q_{S 2}$ & Slow component amplitude & & fitted \\
\hline$\tau_{F 1}$ & Fast fall time & $\sim 650 \mathrm{~ns}$ & fitted \\
\hline$\tau_{F 2}$ & Slow fall time & $\sim 3.2 \mu \mathrm{s}$ & fixed \\
\hline$\tau_{R S}$ & Rise time & $\sim 140 \mathrm{~ns}$ & fixed \\
\hline \multicolumn{4}{|c|}{ Common } \\
\hline$R C$ & Preamp fall time constant & $\sim 6 \mu \mathrm{s}$ & fixed \\
\hline$t_{0}$ & Time offset & $\sim 2 \mu \mathrm{s}$ & fitted \\
\hline$V_{0}$ & Baseline & & fitted \\
\hline
\end{tabular}
with $t_{0}$ being the beginning of the pulse.

The two scintillation components (5) have been introduced to account for the fast and slow decay modes of the $\mathrm{CsI}(\mathrm{Tl})$ crystals. The rise times, $\tau_{R D}$ and $\tau_{R S}$, account for the photodiode, scintillator and the preamp rise times. Overall, the model depends on 11 parameters listed in Table 3 .

Table 3: 11 parameters of the model waveforms.

The preamp fall time constant parameter $R C$ has been determined individually for each chip by selecting the pulses with the fast ionization component alone. The resulting $R C$ constants were smaller than the nominal ones by a few $\mu$ s due to small leakage currents. In order to describe precisely the shapes due to particles stopped in the first photodiode, PD0, both the time constants, $\tau_{R D}$ and $\tau_{F D}$, were fitted and the scintillation components were obviously not used. In case of PD1 and PD2, the rise and fall times $\tau_{R D}$ and $\tau_{F D}$ were fixed. The fits were done using the FUMILI [35] minimization package, a relatively fast and precise implementation of the Gauss-Newton algorithm. Constraining some of the parameters was found inevitable, not only to obtain a good description of the waveforms $\left(\chi^{2}\right)$ but, at the same time, to maintain the global agreement between the reconstructed amplitudes of the three components and the predictions of the range-energy tables (see discussion of Figs. 16, 17.

An additional advantage of using the digitization of the signals and the fitting method, was the knowledge of the actual charges $Q$ for each component, irrespectively of the substantial ballistic deficit (reduction of the amplitude) due to a relatively short discharge time of the preamps $(R C \simeq 6 \mu \mathrm{s})$. For instance, the sum $Q_{S 1}+Q_{S 2}$ represents the total light produced in the 
scintillators. For typical values of the parameters presented in Table 3. the ballistic deficit amounts to about 5-15\% for the ionization signal and to about 25 and $50 \%$ for the fast and slow $\mathrm{CsI}(\mathrm{Tl})$ components, respectively. Usually, the fall time constant of the preamp is a compromise between the level of pileups, the baseline variation, and the ballistic deficit. However, since ballistic deficit is not an issue in our approach, the short preamp fall times, of the order of the time span of the waveform and of the slow CsI(Tl) decay time, have the additional advantage of making the maximum and the tail of the pulse visible within the digitized sample.

Waveforms of low energy particles stopped in the first photodiode, PD0, have been fitted with a single component of the form (4), with the time constant parameters treated as free fit parameters. A more precise time characteristics of the associated current pulse (2) was needed to perform a pulse shape based identification of these particles (see discussion of Fig. 13.

The quality of the fits is demonstrated in Fig. 5. The ordinate represents the measured voltage, in the FADC channels, which is about twice smaller than the actual one due to the matching of the $50 \Omega$ FADC input impedance. The hits corresponding to the selected waveforms are also marked in the identification maps presented in the next section.

Panel A of Fig. 5 shows a waveform for a low energy electron, particle or gamma, registered barely above the acquisition threshold and stopped in PD0 (see also Fig. 13). Here the measured histogram is visible and the deviations from the smooth fit visualize the level of the total noise. The resultant signal distortions, including the photodiode, preamplifier, FADC and pickup noise sources, have been observed on the level of 0.4 $\mathrm{mV}$ rms, corresponding to about $30 \mathrm{keV}$.

Panel B of Fig. 5 presents the quality of the fit with the shape given by an ionization component alone (4), applied to particles stopped in PD0. Its location in the identification map is presented in Fig. 13 This fit allowed for derivation of both, the rise and fall times and, therefore, also of the mode (position of maximum) of the associated current signal (2):

$$
\text { mode }=\frac{\tau_{R D} \tau_{F D}}{\tau_{R D}-\tau_{F D}} \log \frac{\tau_{R D}}{\tau_{F D}}
$$

Panel C of Fig. 5 shows the waveform of a high energy $\alpha$ particle stopped in the CSI 2 crystal. The corresponding hit has been marked in Figs. 7 and 12 .

Panels D-E-F of Fig. 5] show the evolution of the pulse shape of an $\alpha$ particle detected and stopped in the SCT as its energy increases. The corresponding hits have been marked, when possible, in Figs. 6, $8,11$.

The fitting of signals from PD2 (Fig. 5, panel C) produces small artificial ionization contributions for particles which actually do not hit the photodiode (see Fig. 7). It amounts, on average, to $1.7 \pm 0.5 \%$ of the total amplitude. Correspondingly, the artificial contribution of a scintillation component for particles stopped in the PD1 photodiode, and thus producing no light (see representative hit in panel D), is about $3.8 \pm 0.6 \%$. These numbers specify the quality of the pulse shape parametrization and the systematic uncertainty of the decomposition into different components. The artificial scintillation component has

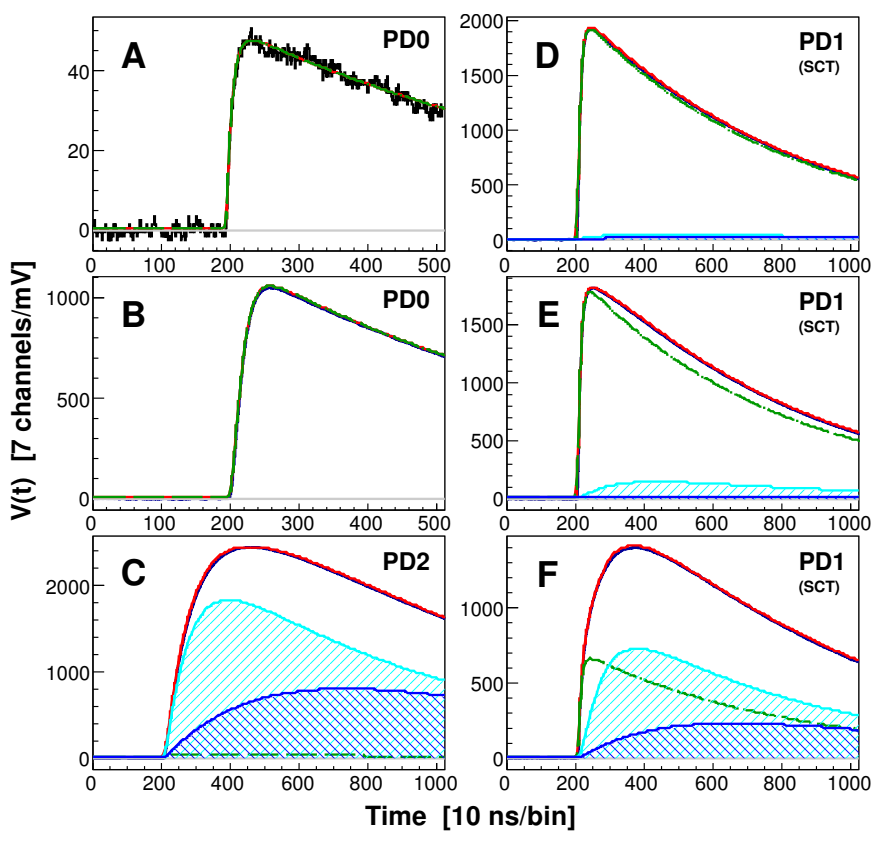

Figure 5: Example waveforms and their decomposition. Full time scale corresponds to 5 (panels A-B) or 10 (panels CF) $\mu \mathrm{s}$. The ordinate represents the measured voltage in the FADC channels. Histogram (when visible): measured waveform. Solid line: sum of all fit components. Dashed line: ionization component. Hatched areas: fast and slow CsI(Tl) scintillation components. A: low-energy electron, particle or gamma ray, barely above the acquisition threshold, stopped in PD0 (see corresponding hit in Fig. 13). B: $\alpha$ particle stopped in PD0 (see Fig. 13). C: $\alpha$ particle stopped in CsI2 (see Fig. 7 , 12). D: $\alpha$ particle stopped in PD1 (see Figs. 6, 8, 9]11). E: $\alpha$ particle barely punching through PD1 and stopped in CsI1 (see Figs. 6, 8, 10, 11). F: higher energy $\alpha$ particle stopped in CsI1 (see Figs. 6, 8, 10, 11,)

been removed by subtracting its well defined fraction from the total amplitude and thus making the ionization and scintillation components of the SCT almost perfectly orthogonal.

\section{Performance}

Figures 613 present various identification (ID) maps obtained by using the parameters of the reconstructed waveform components. The strongest lines in Figs. 6 13 correspond to $p$, $d, t,{ }^{3} \mathrm{He}, \alpha$, and so on, from bottom to top, respectively (see Fig. 16 for precise labeling).

The reconstructed amplitude maps for the first two and the last two photodiodes of the KRATTA module are presented in Figs. 6 and 7

The identification map in Fig. 6 shows a complex spectrum with each "ID-line" composed of two parts: an ordinary SiSi hyperbolic segment at low energies, for particles stopped in PD1, and a more curved part for particles punching through the PD1 photodiode and stopped in the CsI1 crystal. Due to line crossing and its complex structure, this kind of a map cannot 


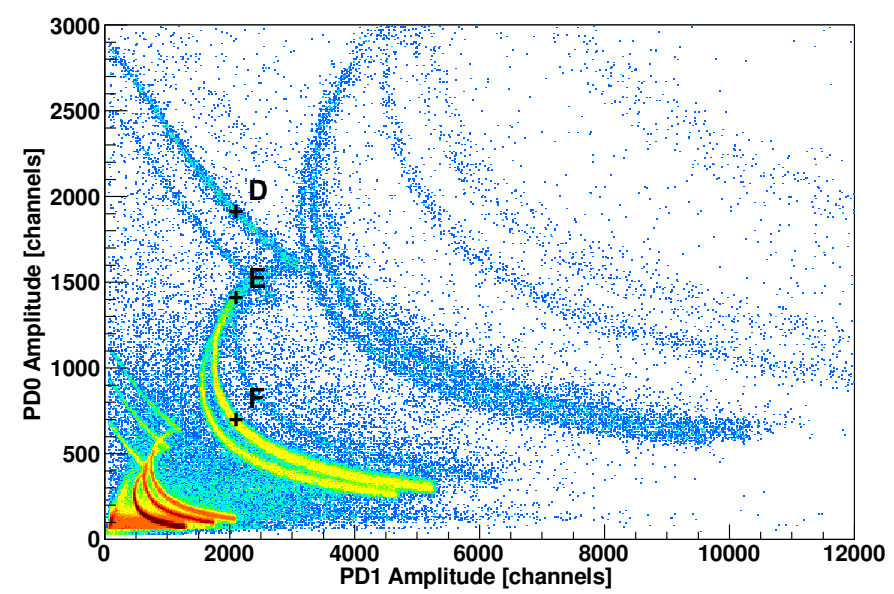

Figure 6: $\triangle \mathrm{E}-\mathrm{E}$ ID map for the first two photodiodes, PD0 vs PD1(SCT), for particles stopped in PD1 or in the thin crystal (CsI1).

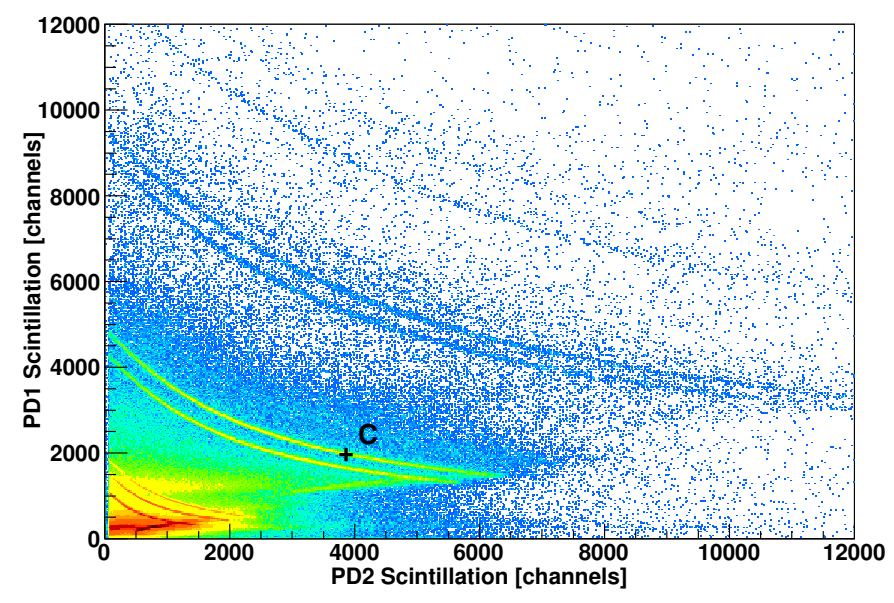

Figure 7: $\triangle \mathrm{E}-\mathrm{E}$ ID map of scintillation signals for CsI1 vs CsI2 detected by PD1 and PD2.

be used for identification, however thanks to the presence of many characteristic punch-through points and curvatures, it is very well suited for energy calibration purposes.

Fig. 7 shows a $\Delta E-E$ map for scintillation signals detected by a second vs third photodiode. Apart from the very good isotopic resolution, one can see a substantial background from the secondary reactions/scatterings in the crystals and also from particles crossing the module at an angle and not originating from the target, as well as back-bendings corresponding to particles punching through the thick crystal (see discussion at the end of this section). Thanks to the pulse shape decomposition, the ionization component for particles hitting the photodiode (nuclear counter effect) at the back of the thick crystal has been easily removed.

Using the individual reconstructed amplitudes for the ionization and for the scintillation components, the map of Fig. 6 can be decomposed into the PD0-PD1(Si) and PD0-PD1(CsI) components of the SCT segment (Figs. 9] and 8). This makes effectively KRATTA even a four-fold telescope.

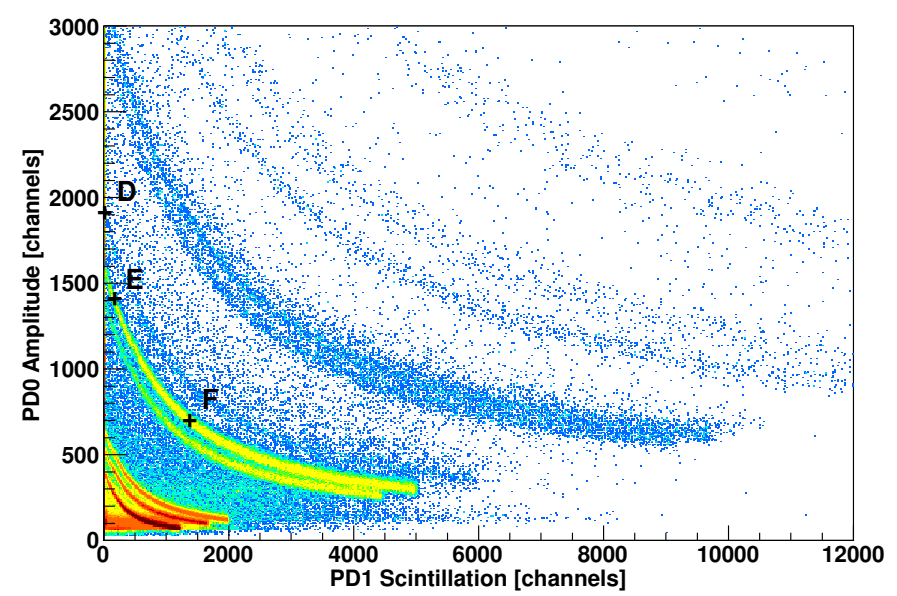

Figure 8: Decomposition of the map from Fig. 6. PD0 vs scintillation detected by PD1 - for particles punching through PD1 and stopped in CsI1.

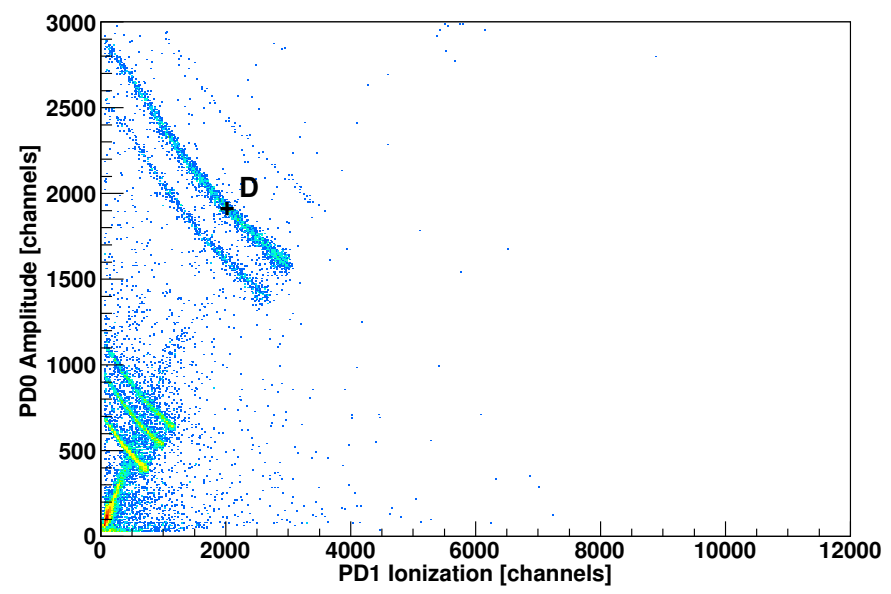

Figure 9: Decomposition of the map from Fig. 6. PD0 vs ionization signal from PD1 - for particles stopped in PD1 (producing no light).

The isotopic resolution visible from Fig. 8 can be improved by summing up the reconstructed ionization components from PD0 and PD1, and thus, increasing the effective thickness of the first $\Delta \mathrm{E}$ layer to $1 \mathrm{~mm}$ of Si. See also inset in Fig. 14 Fig. 11 presents a classical $\Delta$ E-E ID map obtained from Fig. 6 thanks to the pulse shape decomposition (see discussion of Fig. 17 for more details on this transformation and Fig. 14 for the corresponding mass resolution.)

Figures 10 and 12 show the "Fast-Slow" ID maps for the CsI1 and CsI 2 crystals, respectively. The latter represents a variant of the standard representation, using the total light vs the ratio Slow over Fast (see e.g. [36]). The Fast-Slow representation is very useful in many respects in addition to the standard $\Delta \mathrm{E}-\mathrm{E}$ one: One can observe a clear double alpha line ( $\alpha-\alpha$ in Figs. 10 and 12 which is hidden in the $\Delta \mathrm{E}-\mathrm{E}$ representation behind the Li isotope lines. The Fast-Slow map shows a clear " $\gamma$-line" a strong line below the proton line due to $\gamma$-rays (see inset in Fig. 10, which can be precisely isolated and removed from the 


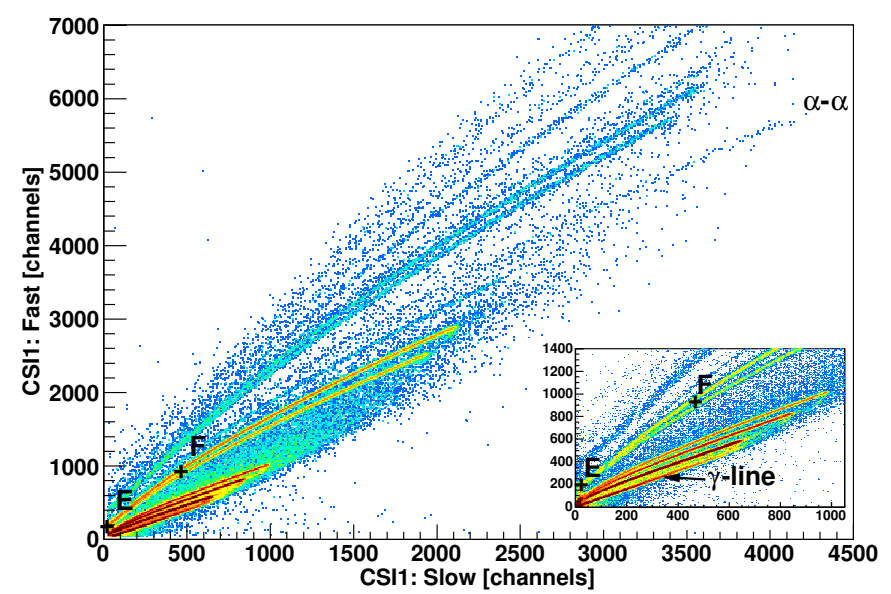

Figure 10: Fast vs Slow components of the scintillation in CsI1. The inset shows more clearly a $\gamma$-line and the $p, d, t$ lines.

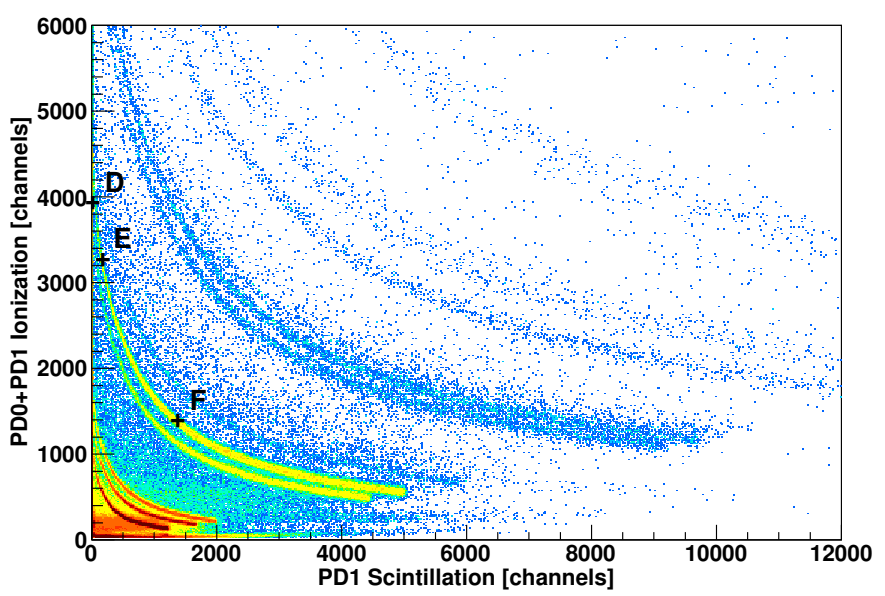

Figure 11: Summed PD0+PD1 ionization components vs scintillation in PD1 for particles stopped in CsI1. Note a slightly improved resolution compared to Fig. 8 (see inset in Fig. 14)

$\Delta \mathrm{E}-\mathrm{E}$ map. Last, but not least, as can be seen from Fig. 12, the punch through segments do not cross the lower lying isotope lines in a way they do in case of the $\Delta \mathrm{E}-\mathrm{E}$ maps (Fig. 7). Thus, the Fast-Slow maps allow to isolate the punch through segments in a much more precise way. Unfortunately, the punch through segments eventually merge with the $\gamma$-lines, which makes it difficult to discriminate between these two.

Figure 13 demonstrates yet another powerful feature of the pulse shape analysis. It allowed to perform an identification of the majority of light particles stopped in the first photodiode by plotting the amplitude vs mode of the reconstructed current signal (6). Due to the constant value of the field within the intrinsic region of the PIN diode the enhancement of the resolution for stopped particles is not expected to be as pronounced as in the case of reverse mount PN detectors [37], nevertheless, the relation between the range and the carrier collection time seems to be still strong enough to enable the isotopic separation of light particles. This method allowed to reduce the lower identification threshold, due to the thickness of the first photodiode, (see

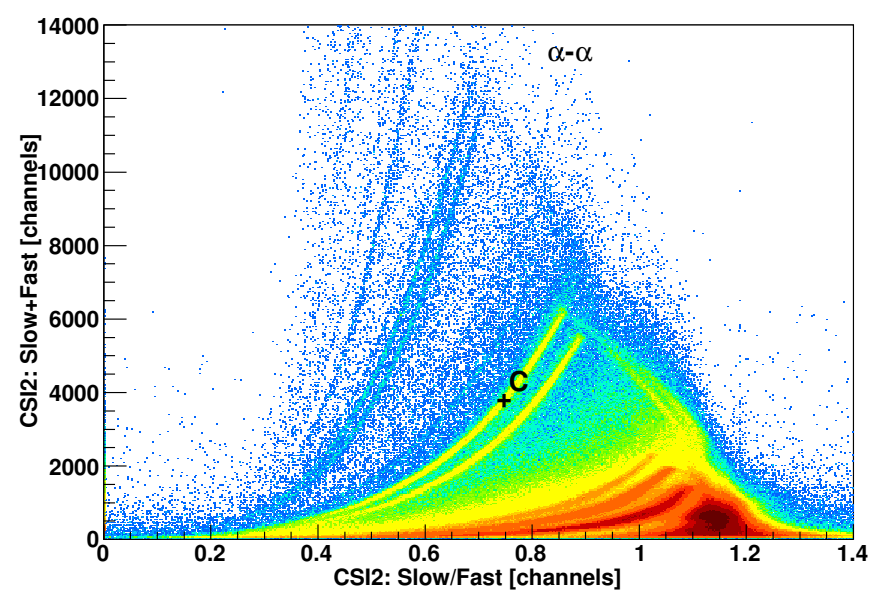

Figure 12: Total light vs Slow over Fast components of the scintillation in CsI2. Note, that unlike in Fig. 7) the punch through lines do not cross the lower lying isotope lines.

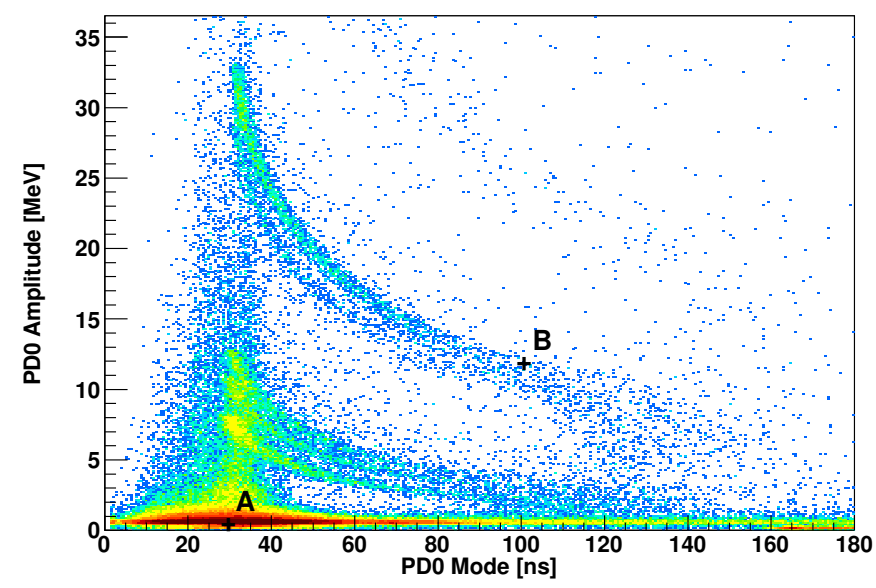

Figure 13: Amplitude vs mode of the current signal for particles stopped in PD0, obtained from a single Si chip.

Table 2) from 8.3 to about $2.5 \mathrm{MeV}$ for protons, where they are still resolvable from deuterons (see two bottom lines in Fig. 13). This effectively corresponds to the reduction of the thickness of the first $\Delta \mathrm{E}$ layer from 500 to about $65 \mu \mathrm{m}$ of $\mathrm{Si}$.

One should stress, that the time scale presented in Fig. 13 exceeds by about a factor of 3 the collection times estimated on the basis of the mobilities of the carriers alone, thus the plasma delay, or other effects, seem to be quite important in slowing down the collection process.

The mass resolution for particles stopped in the thin crystal (CsI1) and in the thick one (CsI2) can be viewed from Figs. 14 and 15 , respectively.

The background in Fig. 14, resulting mainly from the secondary reactions/scattering in the crystal, amounts to about $6 \%$ for the hits below the $p, d, t$ peaks. For energetic $Z=1$ particles traversing the thick crystal $(12.5 \mathrm{~cm}$ of CsI) the secondary reaction probability amounts to about $46 \%$ (Fig. 15). These probabilities agree reasonably well with the simple estimates obtained using the nuclear collision length of $22.30 \mathrm{~cm}$ for CsI 


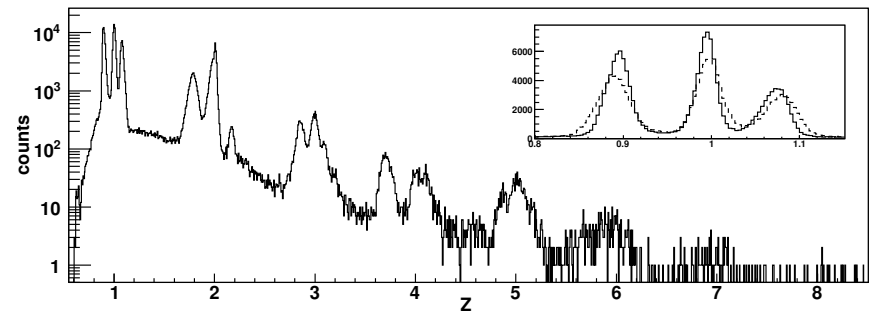

Figure 14: Particle identification spectrum (charge distribution) obtained from the map of Fig. 11, for particles stopped in the thin crystal. The inset shows the $p, d, t$ peaks (in linear scale) obtained from the Fig. 11 (solid) and Fig. 8 (dashed histogram), respectively.

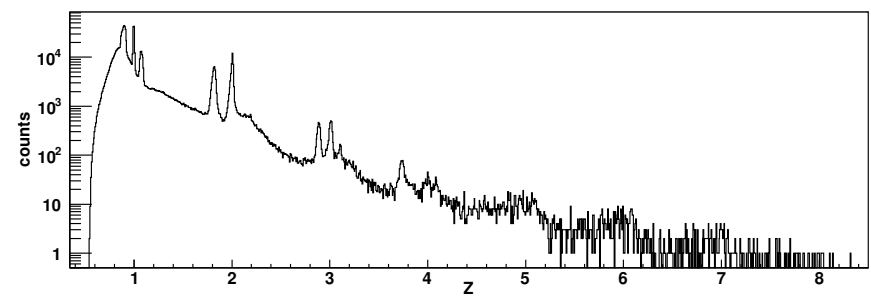

Figure 15: Same as Fig. 14, but obtained from the map of Fig. 7. for particles stopped in the thick crystal.

[38], which yields the probabilities of $11 \%$ and $43 \%$ for 2.5 and $12.5 \mathrm{~cm}$ of CsI, respectively. The background measured under the $p, d, t$ lines includes also some contribution from the secondary reactions of neutrons and heavier charged particles, as well as from the accidental coincidences. The $\gamma$ and punchthrough hits have been removed from the background, in both cases. The high secondary interaction probability obviously deteriorates the identification quality and defines the limits for applying the telescope method to intermediate energies.

\section{Discussion and remarks}

Since the parametrization of the pulse shapes is only approximate and has no deep physical background, one can ask how precise is the decomposition into individual components and how physical they are. In order to address these questions, the $\triangle \mathrm{E}-\mathrm{E}$ map of the SCT (PD1+CsI1) has been compared to the predictions of the ATIMA range-energy tables. Such a comparison requires the knowledge of the energy calibration of both, the silicon photodiodes and of the CsI(Tl) light output. The calibration has been performed using the ID map of Fig. 6 (see Fig. 16) which is relatively insensitive to the details of the decomposition and is perfectly suited for the energy calibration due to its richness in characteristic punch-through points and curvatures.

The calibration routine allowed to adjust the thicknesses of the dead and active layers, the energy calibration parameters, as well as the light-energy conversion parameters. For the latter, a simple integrated Birks' formula (see e.g. [34]) has been used, with $d E / d x \propto A Z^{2} / E$, yielding a commonly applied two-

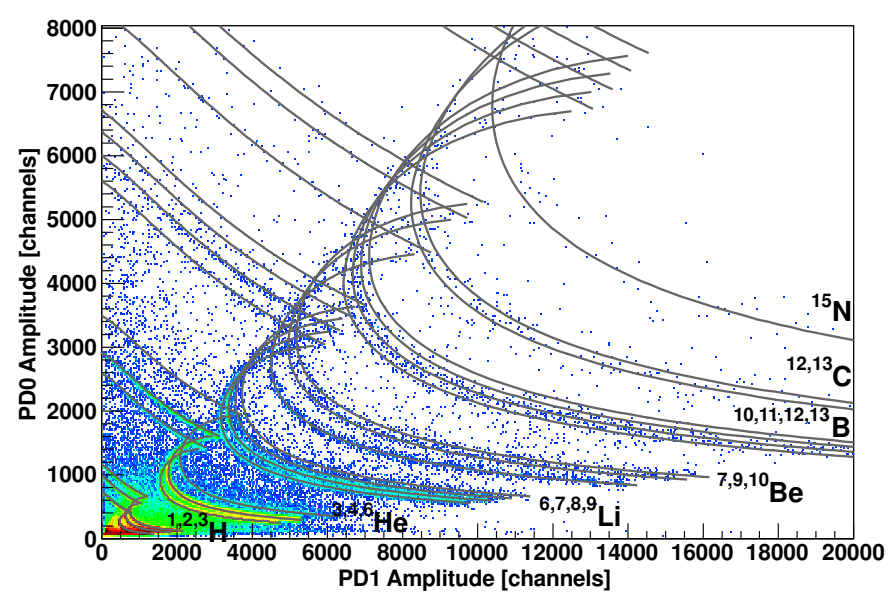

Figure 16: Same as Fig. 6 but with the superimposed ID lines calculated using the ATIMA range-energy tables.

parameter Light-Energy relation [39], applicable for particles stopped in the scintillator:

$$
\text { Light }=a_{1}\left(E-a_{2} A Z^{2} \log \left[1+\frac{E}{a_{2} A Z^{2}}\right]\right)
$$

with $E$ being the energy, $A, Z$ - mass and atomic numbers of the stopped particle, $a_{1}, a_{2}$ - the gain and quenching parameters.

Having this (inverse) calibration it was possible to superpose the ATIMA lines on the decomposed ID map for the Single Chip Telescope segment of the KRATTA module. The result is presented in Fig. 17 and is worth a comment.

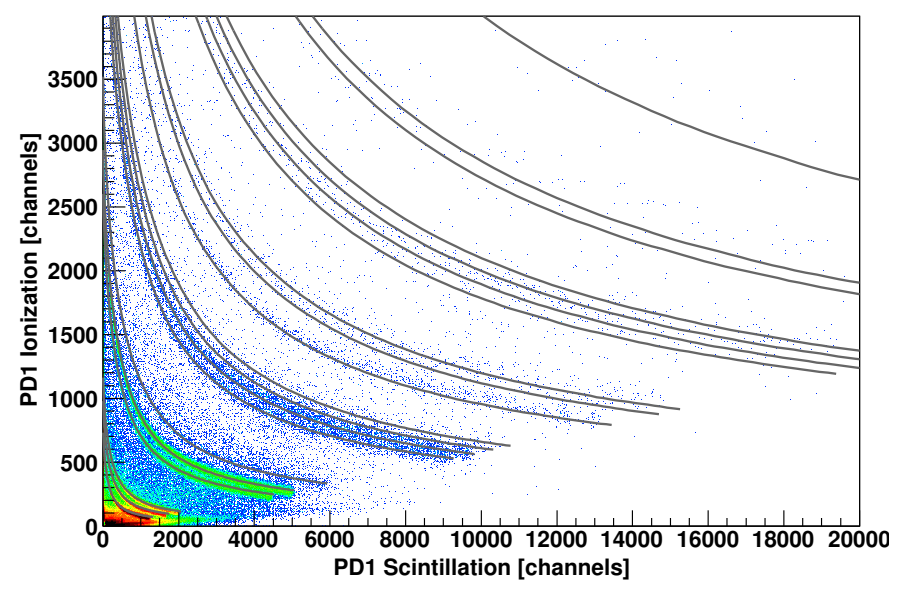

Figure 17: Decomposed SCT $\triangle \mathrm{E}-\mathrm{E}$ identification map (obtained with a single readout channel) with the superimposed ID lines calculated using the ATIMA tables. The sequence of lines is the same as in Fig. 16.

The observed very good agreement between the reconstructed amplitudes and the range-energy table predictions is quite non-trivial taking into account the simplicity of the pulse shape parametrization. In order to obtain this kind of agreement, which is still a compromise, it was not enough to set all 
the parameters free and search for the minimum of the $\chi^{2}$ distribution. Such an automatic procedure guarantees the best fit to the waveforms, but not necessarily guarantees the follow up of the trends defined by the range-energy tables. In general, an automatic procedure can lead, depending also on the starting values, to some local minima, produce discontinuities or lead in directions diverging from the ATIMA lines. The presented agreement has been obtained after searching for the optimal values and fixing some of the parameters (see Table 3). The agreement with the ATIMA lines could be made even better, for instance by freeing the $\tau_{R S}$ parameter, however at the expense of loosing completely the mass resolution in Fast-Slow representation (contrary to the case presented in Fig. 10). Thus, the presented agreement is a result of an iterative procedure of fixing some of the parameters and also of the energy calibration parameters, but once the crucial parameters are constrained the fitting proceeds automatically.

The whole procedure could probably be better automatized by using more realistic pulse shape parametrizations, especially for individual electron and hole components of the ionization signal, taking into account the plasma delay effects, interactions between carriers, diffusion effects, etc [33]. However, to our knowledge, such pulse shape parametrizations are still to be developed. Definitely, a more realistic preamplifier response function would improve the resolution and would allow for disentangling between physical and instrumental parameters. A more realistic parametrization and analysis would be worth the effort of implementing it to e.g. better understand the relation between the range and the charge collection times which enable the identification of the particles stopped in the silicon detectors. Nevertheless, any more sophisticated analysis would definitely slow down even more the analysis.

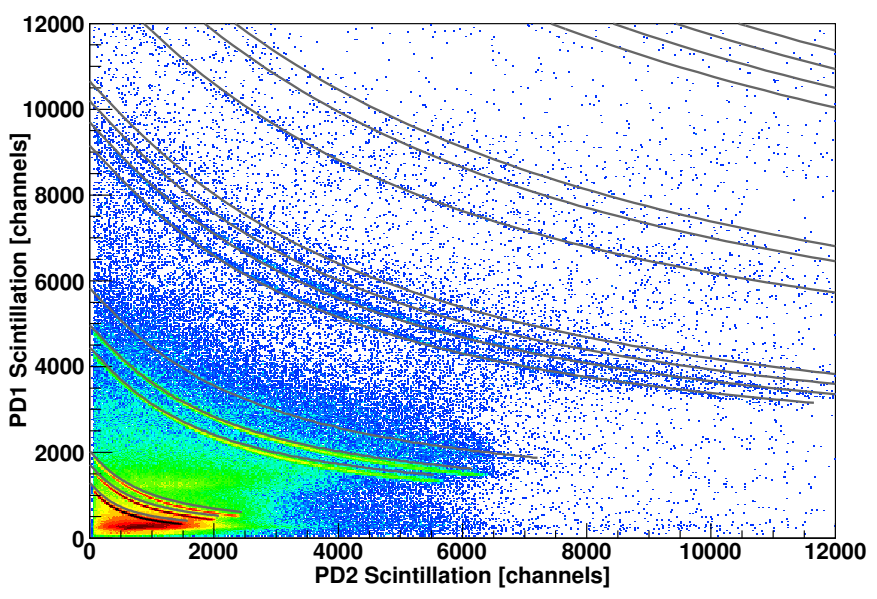

Figure 18: $\Delta \mathrm{E}-\mathrm{E}$ identification map for scintillation signals from CsI1 vs CsI2, with the superimposed ID lines calculated using the ATIMA tables. The sequence of lines is the same as in Fig. 16 The $\gamma$-line and punch-through hits have been removed (cf. Fig. 7).

Finally, Fig. 18 shows the calculated ATIMA lines superimposed on the $\Delta \mathrm{E}-\mathrm{E}$ identification map for scintillation signals from thin vs thick $\mathrm{CsI}(\mathrm{Tl})$ crystals. The slight overestimation of the $\Delta \mathrm{E}$ component for $Z=1$ particles results most probably from the simplicity of the Light-Energy conversion formula (7) and (or) from the light output non-uniformity of the crystals. The overall agreement is, nevertheless, quite satisfactory and the calculated lines can be used not only to derive the energy calibration parameters, but also for identification (see Fig. 15, after small manual adjustments.

The energy calibration routine based on the ATIMA rangeenergy tables operated on all three maps (Figs. 16 18) simultaneously, allowing for a consistent determination of the calibration parameters for all photodiodes and both crystals. Specifically, for the $\mathrm{CsI}(\mathrm{Tl})$ crystals with 1500 ppm of $\mathrm{Tl}$ concentration, the quenching parameter $a_{2}$ was found to be equal to 0.32 , a value compatible with the one from [39] and about $20 \%$ larger than the average one quoted for the INDRA crystals [40]. The calibration of the SCT allowed also to estimate the efficiency of the scintillation. The combination of a relatively high $\mathrm{Tl}$ doping, high reflectance of the wrapping and large active area of the photodiode, resulted in a relatively high efficiency for energy-light conversion. It was found that only about 6 times more energy was needed to produce an electron-hole pair in the photodiode through a scintillation process in the CsI(Tl) crystal than in the direct ionization process in the photodiode. This fact is worth noting, since for the "good scintillators" quoted in [34] this factor amounts to 15-20.

Obviously, a drawback of the telescope method at high energies, is the high level of the secondary reactions. In order to handle this problem, a more sophisticated methods, neural networks and discriminant analysis are being tested, but this goes beyond the scope of this article.

\section{Summary}

A new, low threshold, broad energy range, versatile array of triple telescopes, KRATTA, has been constructed. The modules, equipped with digital electronics chains, allowed for isotopic identification of light charged reaction products.

Pulse shape analysis allowed for realistic decomposition of the complex SCT pulse shapes into individual ionization and scintillation components and eventually profit from the, otherwise harmful, nuclear counter effect. The isotopic resolution obtained using a single readout channel was found to compete very well with those obtained using the standard two channel readout. The applied pulse shape analysis permitted also the identification of particles stopped in the first photodiode and the reduction of the identification threshold, due to the thickness of the first photodiode, by a factor of three. Thanks to the pulse shape analysis, it was also possible to obtain the ballistic deficit free amplitudes, which allowed for easy energy calibration and identification based on the predictions of the range-energy tables.

The array has met the expectations, fulfilled the design requirements and performed very well during the ASY-EOS experiment at GSI. 


\section{Acknowledgments}

Work made possible through funding by Polish Ministry of Science and Higher Education under grant No. DPN/N108/GSI/2009.

We (S.K.) acknowledge the support by the Foundation for Polish Science - MPD program, co-financed by the European Union within the European Regional Development Fund.

Stimulating discussions, expertise and help of Giacomo Poggi as well as of Marian Pârlog, Remi Bougault and Hector Alvarez Pol during the design and test phase of the prototypes are gratefully acknowledged.

\section{References}

[1] Bao-An Li et al., Phys. Rep. 464 (2008) 113.

[2] M. Di Toro et al., J. Phys. G 37 (2010) 083101.

[3] R.T. de Souza et al., Eur. Phys. J. A 30 (2006) 275.

[4] J.B.A England, "Techniques in Nuclear Structure Physics", Wiley (1974).

[5] R.W. Leo, "Techniques for Nuclear and Particle Physics Experiments", Springer-Verlag (1987).

[6] R. Bougault et al., Nucl. Instrum. Methods A 259 (1987) 473.

[7] I. Iori et al., Nucl. Instr. Meth. A 325 (1993) 458

[8] S.P. Avdeyev et al., Nucl. Instrum. Methods A 332 (1993) 149.

[9] J. Pouthas et al., Nucl. Instr. Meth. A 357 (1995) 418.

[10] K. Kwiatkowski et al., Nucl. Instr. Meth. A 360 (1995) 583.

[11] F. Gramegna et al., Nucl. Instr. Meth. A 389 (1997) 474.

[12] M. Bini et al., Nucl. Instr. Meth. A 515 (2003) 497.

[13] F. Gimeno-Nogues et al., Nucl. Instr. Meth. A 399 (1997) 94

[14] B. Davin et al., Nucl. Instr. Meth. A 473 (2001) 302.

[15] A. Pagano et al., Nucl. Phys. A 734 (2004) 504.

[16] M.S. Wallace et al., Nucl. Instr. Meth. A 583 (2007) 302.

[17] S. Wünschel et al., Nucl. Phys. A 604 (2009) 578.

[18] http://fazia2.in2p3.fr/spip/

[19] P. Russotto et al., IWM 2011 Proc., Conf. Proc. Vol. 105, p. 91. P. Russotto et al., IWM 2009 Proc., Conf. Proc. Vol. 101, p. 22. http:// www.irb.hr/users/mkis http://www.ct.infn.it/asyeos2010 P. Russotto et al., Phys. Lett. B 697 (2011) 471.

[20] Th. Blaich et al., Nucl. Instr. Meth. A 314 (1992) 136. P. Pawłowski et al., Nucl. Instr. Meth. A 694 (2012) 47.

[21] A. Schüttauf et al., Nucl. Phys. A 607 (1996) 457.

[22] A. Budzanowski et al., Nucl. Instr. Meth. A 482 (2002) 528.

[23] HAMAMATSU Si Photodiode for Direct Detection (S5377-0052(X)).

[24] Manufacturer: Institute of Modern Physics, Chinese Academy of Sciences, Lanzhou, China.

[25] G. Pasquali et al., Nucl. Instr. Meth. A 301(1991) 101

[26] 3M Vikuiti ${ }^{\mathrm{TM}}$ ESR foil, recommended by the crystal manufacturer.

[27] http://www-linux.gsi.de/ weick/atima

[28] Z. Sosin et al., in preparation.

[29] CAEN V1724 digitizer.

[30] http://www.gsi.de/informationen/wti/ee/elekt_ entwicklung/triva_e.html

[31] RIO4-8072RE, CES, http://www.ces.ch

[32] http://www-win.gsi.de/daq/

[33] C.A.J. Ammerlaan et al., Nucl. Instr. Meth. 22 (1963) 189. A. Alberigi Quaranta et al., Nucl. Instr. Meth. 57 (1967) 131. G. Pausch et al., Nucl. Instr. and Meth. A 337 (1994) 573. M. Pârlog et al., Nucl. Instr. Meth. A 613 (2010) 290. Z. Sosin, Nucl. Instr. Meth. A 693 (2012) 170.

[34] G.F. Knoll, "Radiation Detection and Measurement", 4-th ed., Wiley (2010), Chapt. 9, 16.

[35] http://root.cern.ch A.J. Ketikian et al., Nucl. Instr. and Meth. A 314 (1992) 578. S.N. Dymov et al., Nucl. Instr. and Meth. A 440 (2000) 431.

[36] F. Benrachi et al., Nucl. Instr. and Meth. A 281 (1989) 137.

[37] G. Pausch et al., Nucl. Instr. and Meth. A 365 (1995) 176. L. Bardelli et al., Nucl. Instr. and Meth. A 654 (2011) 272.

[38] http://pdg.lbl.gov/2012/AtomicNuclearProperties/HTML_ PAGES/141.html
[39] D. Horn et al., Nucl. Instr. and Meth. A 320 (1992) 273.

[40] M. Pârlog et al., Nucl. Instr. and Meth. A 482 (2002) 693. 